\title{
A Logic Architecture for 360 ADAS-Alerts for Hazards Detection Based in Driver Actions
}

\author{
Javier Izquierdo-Reyes ${ }^{1}$, Luis A. Curiel-Ramirez ${ }^{1}$, Ricardo A. Ramirez-Mendoza ${ }^{1}$ and M. Rogelio Bustamante-Bello ${ }^{1}$ \\ ${ }^{1}$ Escuela de Ingeniería y Ciencias, Tecnológico de Monterrey \\ Calle del Puente 222, Tlalpan, Ejidos de Huipulco, 14380 Ciudad de México, México.
}

\begin{abstract}
In this work is presented a novel approach for passive safety in vehicles by Advanced Driver Assistance Systems (ADAS) alert emission in $360^{\circ}$ around driver to notify about hazards nearby the vehicle depending on the actions taken by driver per the context. This proposal would create a more robust system compared to current passive ADAS systems since the feedback to driver is in the same direction that hazard is detected (Punctual Sound Source Alert), compared with most assistance systems that emits sounds from the monitor or from the dashboard provoking distractions when emits alerts unnecessarily. The increase of security by this method will allow the driver to be aware of their surroundings even in a very quiet cabin or in a noisy environment. Also, it would detect the steering wheel angle, speed of movement and the activation of turning lights among other alerts, which would allow us to define a critical action during driving; apart from using sensors and cameras aimed at the driver to detect patterns of movement during these critical actions and have a prediction of a possible turn or manoeuvre when driving, refer to Figure 1. It will be necessary a reconfiguration of the alert in frequency, time of action depending upon the level of risk to prevent an accident or to reduce the consequences in an imminent accident.
\end{abstract}

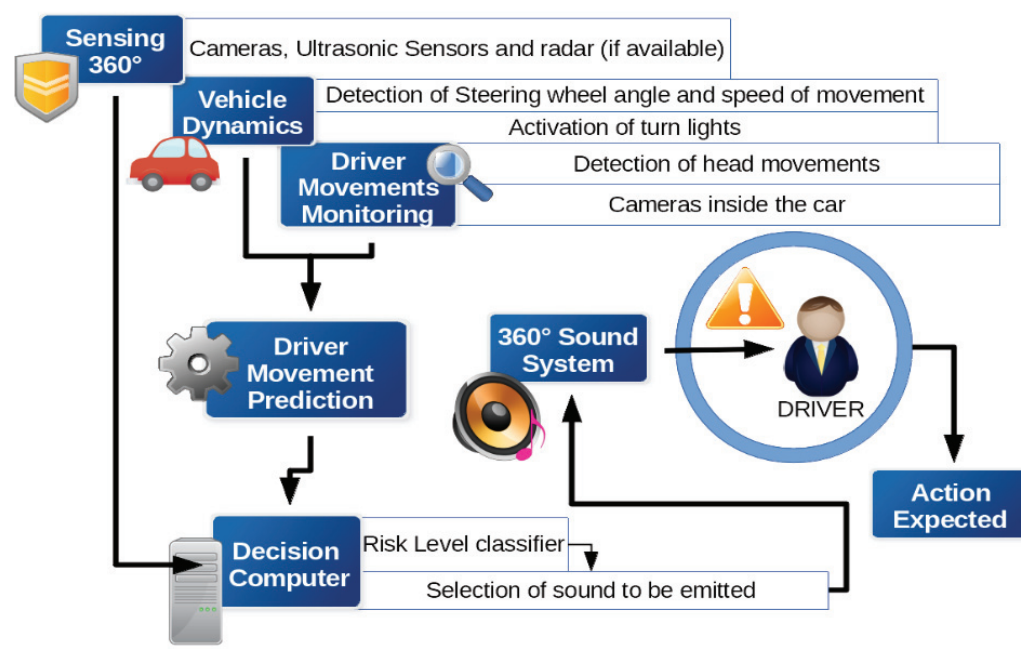

Figure 1. Graphical Abstract.

\section{Introduction}

Advanced driver assistance systems are becoming more common in mid-segment vehicles and are fully required in the high segment due to high added value. But despite all the new developments, the issuance of alerts has not evolved beyond a screen.

Emission alert is necessary to avoid traffic accidents, however, not all alerts are well perceived by all the people, or sometimes the screen is overloaded and therefore induces a state of distraction.

Nowadays there are different kind of alerts such as:

- Sound

- Visual

- Audio-visual

- Haptics

In Table 1 is a list of brands and models that incorporates some passive ADAS that emit this type of alerts. 
In previous studies [1], [2], have been demonstrated that a poorly designed alert can be worse than if no alert is emitted because of the distraction it will produce, in [3] we used electroencephalography to detect driver concentration, and we found that some drivers got distracted due to ADAS Alerts.

Also, the National Highway Traffic Safety Administration (NHTSA) of the United States of America (USA) has conducted some studies to analyse the effects that auditory alerts have over the driver and how they are perceived (Warnings, Alerts, Status Notification or Social Notification) [4]. This research has been done to promote a standardized set of sound that allows easily and clearly identify urgent alert from other messages.

On the other hand, the proposed vibration and audio alerts have been shown to reduce reaction time significantly, even if the driver is involved in a second task, such as a telephone call, a high working memory load task [2], [5], [6]

Table 1. Visual or sound alerts in ADAS in Latin-American

\begin{tabular}{|c|c|c|c|c|c|c|c|c|c|}
\hline Brand & Models & $\begin{array}{l}\text { Included } \\
\text { Since } \\
\text { (Year) }\end{array}$ & $\begin{array}{c}\text { Driver } \\
\text { drowsines } \\
\text { s alert }\end{array}$ & $\begin{array}{l}\text { Lane } \\
\text { keep } \\
\text { assist }\end{array}$ & $\begin{array}{l}\text { Traffic } \\
\text { Alert }\end{array}$ & $\begin{array}{c}\text { Speed } \\
\text { limit alert }\end{array}$ & $\begin{array}{l}\text { Blind spot } \\
\text { alert }\end{array}$ & $\begin{array}{c}\text { Pre- } \\
\text { collision } \\
\text { System }\end{array}$ & $\begin{array}{c}\text { Parking } \\
\text { Sensor } \\
\text { alerts }\end{array}$ \\
\hline $\begin{array}{c}\text { Toyota Motor } \\
\text { Corporation }\end{array}$ & All & 2015 & Optional & Optional & Optional & & $\checkmark$ & Optional & $\checkmark$ \\
\hline \multirow{2}{*}{\begin{tabular}{|c|} 
Hyundai \\
Motor \\
Company \\
\end{tabular}} & Stinger & 2018 & $\checkmark$ & $\checkmark$ & Optional & & $\checkmark$ & $\checkmark$ & $\checkmark$ \\
\hline & All & 2015 & & & & & $\checkmark$ & & Optional \\
\hline \multirow{6}{*}{ VW Group } & Passat & 2013 & $\checkmark$ & & & & $\checkmark$ & & $\checkmark$ \\
\hline & Touareg & 2015 & $\checkmark$ & Optional & & & & Optional & $\checkmark$ \\
\hline & Golf & 2013 & $\checkmark$ & & & & & & $\checkmark$ \\
\hline & A3-6 & 2013 & $\checkmark$ & Optional & Optional & & $\checkmark$ & $\checkmark$ & $\checkmark$ \\
\hline & Leon & 2013 & Optional & Optional & & & & & $\checkmark$ \\
\hline & All & 2013 & Optional & & & & & & Optional \\
\hline \multirow{5}{*}{$\begin{array}{c}\text { General } \\
\text { Motors } \\
\text { Corporation }\end{array}$} & Equinox & 2012 & & Optional & & Optional & $\checkmark$ & Optional & $\checkmark$ \\
\hline & Terrain & 2012 & & Optional & & Optional & $\checkmark$ & Optional & $\checkmark$ \\
\hline & Astra & 2015 & & & & $\checkmark$ & & & $\checkmark$ \\
\hline & Malibu & 2016 & Optional & $\checkmark$ & & & $\checkmark$ & $\checkmark$ & $\checkmark$ \\
\hline & All & 2008 & & & & & & & Optional \\
\hline \multirow{4}{*}{$\begin{array}{l}\text { Ford Motor } \\
\text { Company }\end{array}$} & Mustang & 2013 & Optional & $\checkmark$ & & Optional & $\checkmark$ & $\checkmark$ & $\checkmark$ \\
\hline & Focus & 2017 & Optional & $\checkmark$ & & & $\checkmark$ & $\checkmark$ & $\checkmark$ \\
\hline & Escape & 2016 & Optional & $\checkmark$ & & & $\checkmark$ & $\checkmark$ & $\checkmark$ \\
\hline & All & 2006 & & & & & & & Optional \\
\hline \multirow{3}{*}{$\begin{array}{l}\text { Nissan } \\
\text { Motor }\end{array}$} & Leaf & 2013 & & $\checkmark$ & & & $\checkmark$ & & $\checkmark$ \\
\hline & Infiniti Q/Qx & 2015 & Optional & $\checkmark$ & & & $\checkmark$ & Optional & $\checkmark$ \\
\hline & All & 2013 & & & & & Optional & & Optional \\
\hline \multirow{3}{*}{$\begin{array}{l}\text { Fiat Chrysler } \\
\text { Automobiles }\end{array}$} & Pacifica & 2017 & $\checkmark$ & $\checkmark$ & & & $\checkmark$ & $\checkmark$ & $\checkmark$ \\
\hline & 300 & 2016 & $\checkmark$ & $\checkmark$ & & & $\checkmark$ & $\checkmark$ & $\checkmark$ \\
\hline & All & 2010 & & & & & & & Optional \\
\hline \multirow{4}{*}{$\begin{array}{c}\text { Honda } \\
\text { Motor } \\
\text { Company }\end{array}$} & Accord & 2016 & $\checkmark$ & Optional & Optional & & $\checkmark$ & & $\checkmark$ \\
\hline & RDX & 2016 & Optional & $\checkmark$ & & & $\checkmark$ & $\checkmark$ & $\checkmark$ \\
\hline & RLX & 2013 & Optional & $\checkmark$ & & & $\checkmark$ & & $\checkmark$ \\
\hline & All & 2010 & & Optional & & & Optional & & Optional \\
\hline \multirow{2}{*}{$\begin{array}{c}\text { Suzuki } \\
\text { Motor } \\
\text { Corporation }\end{array}$} & Vitara & 2015 & Optional & Optional & & & $\checkmark$ & $\checkmark$ & $\checkmark$ \\
\hline & All & 2013 & & & & & Optional & Optional & Optional \\
\hline \multirow{2}{*}{$\begin{array}{c}\text { Grupo } \\
\text { PSA }(\text { Peugeot } \\
)\end{array}$} & 3008 & & Optional & $\checkmark$ & & & $\checkmark$ & $\checkmark$ & $\checkmark$ \\
\hline & All & 2011 & & & & & Optional & Optional & Optional \\
\hline \multirow{3}{*}{ Renault } & Clio & 2017 & Optional & Optional & & & $\checkmark$ & Optional & $\checkmark$ \\
\hline & Koleos & 2017 & $\checkmark$ & Optional & & & $\checkmark$ & Optional & \\
\hline & All & 2012 & & & & & Optional & & Optional \\
\hline \multirow{2}{*}{$B M W A G$} & $4,5,6$ & 2014 & Optional & $\checkmark$ & & & $\checkmark$ & $\checkmark$ & $\checkmark$ \\
\hline & All & 2010 & Optional & Optional & Optional & Optional & $\checkmark$ & Optional & $\checkmark$ \\
\hline \multirow{2}{*}{$\begin{array}{c}\text { Mazda Motor } \\
\text { Corporation }\end{array}$} & 6 & 2017 & Optional & & & & $\checkmark$ & & $\checkmark$ \\
\hline & MX-5 & 2016 & & Optional & & $\checkmark$ & $\checkmark$ & $\checkmark$ & $\checkmark$ \\
\hline
\end{tabular}




\begin{tabular}{|c|c|c|c|c|c|c|c|c|c|}
\hline & All & 2012 & & & & & Optional & & $\checkmark$ \\
\hline \multirow{2}{*}{$\begin{array}{c}\text { Mitsubishi } \\
\text { Motors } \\
\text { Corporation }\end{array}$} & outlander & 2015 & Optional & Optional & & & $\checkmark$ & & $\checkmark$ \\
\hline & All & 2012 & & & & & Optional & & Optional \\
\hline \multirow{2}{*}{$\begin{array}{l}\text { Tata Motors } \\
\text { Limited } \\
\text { (Jaguar, } \\
\text { Land Rover) }\end{array}$} & $\mathrm{xj} / \mathrm{xf}$ & 2014 & Optional & $\checkmark$ & & Optional & $\checkmark$ & $\checkmark$ & $\checkmark$ \\
\hline & All & 2012 & & Optional & Optional & & $\checkmark$ & Optional & $\checkmark$ \\
\hline \multirow{2}{*}{$\begin{array}{c}\text { Geely } \\
\text { Automobile } \\
\text { (Volvo) }\end{array}$} & S90 & & $\checkmark$ & $\checkmark$ & & Optional & $\checkmark$ & $\checkmark$ & $\checkmark$ \\
\hline & All & 2011 & Optional & Optional & & & Optional & Optional & $\checkmark$ \\
\hline \multirow{2}{*}{$\begin{array}{c}\text { Daimler } \\
\text { AG(Mercede } \\
\text { s-Benz) }\end{array}$} & GLA gamma & 2012 & $\checkmark$ & Optional & & Optional & $\checkmark$ & $\checkmark$ & $\checkmark$ \\
\hline & All & 2011 & & & & & Optional & & $\checkmark$ \\
\hline
\end{tabular}

\section{Alert implications}

Distraction: As stated above, if the alert system is not precisely designed, it may involve distraction during driving due to confusion about the type of alert or risk detected, which generates a high mental workload and usually means a change in the attention of the road to dashboard or infotainment system [7].

Modern vehicle security systems display information on a central screen that is sometimes overloaded due to the amount of information being displayed to the user (music, GPS, social media, application system status, alerts, etc.).

It is because of the alerts are difficult to understand and represent a risk due to the need to change the look from the road to the screen to confirm the source of the alert.

False Alarm: Another problem with ADAS systems is when a false alarm is emitted, it is more common in systems based on artificial vision, which have a certain percentage of precision, which allows errors. Or when the alert is too repetitive due to an incorrect configuration and sounds at all times. All these false alarms are annoying to the driver and create a lack of confidence in the system [8]-[10].

In addition, a false negative is a big problem especially for vehicles on the scale 2 or higher automation by NHTSA [10], as these vehicles can be 'autonomous' under certain conditions. The best example was in 2015, when, unfortunately, a false negative cost the life of at least one person in the USA. Because the system was not able to detect a truck, the car did not stop and kill the passenger.

So, to avoid potential dangers created by the warnings and alerts in non-autonomous cars, it is important to design the alerts in the best way or making them adaptable to the driver.

\section{Perception of alerts}

According to the research made by Westat and the NHTSA [4], the perception of alerts and the means of urgency depends strongly of the noise condition and the urgency of the alert.

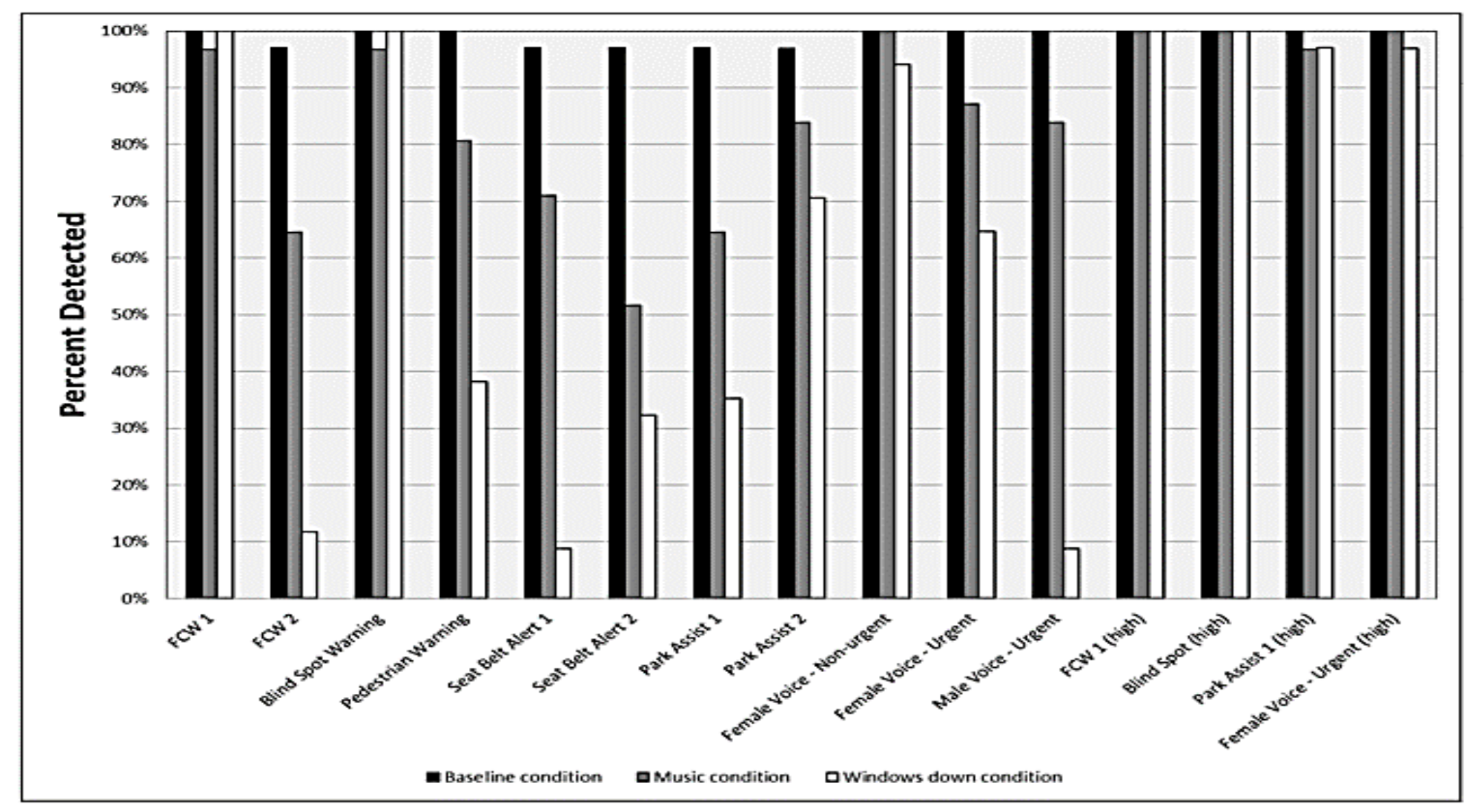

Figure 2. Percentage of drivers who detects alerts [4]. 
The configuration for each alert is indicated and explained in detail in the experimental protocol of the Study 2 'Warning perception in ambient noise environments' [4]

In figure 2 and 3 are shown the results obtained in an experiment [4] with 34 drivers, in which can be observed that low frequencies are less detectable than high in noise conditions, for example, in Figure 1. The FCW2, Seat Belt alert 1 and male voice has $11 \%$ or less in percentage detected for the windows down condition, while the FCW, Blind spot Warning, FCW1 (high), and Blind Spot (high) reached the $100 \%$ of detection.

And the same happens with the mean urgency rating for FCW2 and Seat Belt Alert 1.

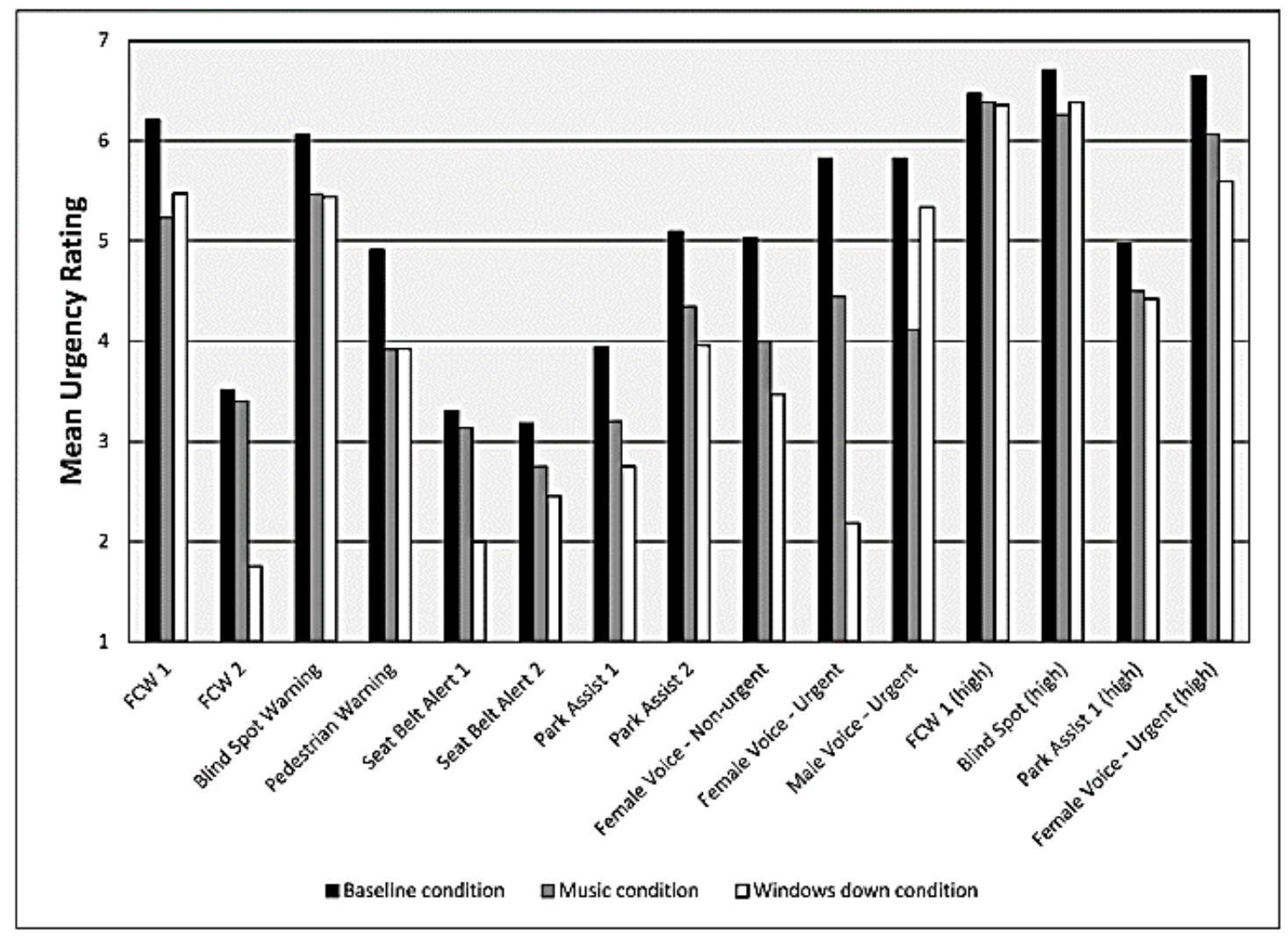

Figure 3. Mean Urgency Rating [4], [11].

For the male voice is different and drivers rated relative high the mean urgency, it is possible, because speech warnings are fast to be classified and better understood than bursts or beeps.

To explain the effect Zhang et. al. [1] developed a mathematical model in which is analysed the response time due to speech alerts and all aspects involved in the process.

\section{$4360^{\circ}$ ADAS alert proposal}

After analysing the state of the art of the alerts, the variety of them and the experience obtained in the experiment developed in [3] and [12], we propose the use of a surround system $360^{\circ}$ inside the vehicle as is shown in Figure 4, to give the user the ability to know the existence of hazards around him even without the need of see a monitor or infotainment screen.

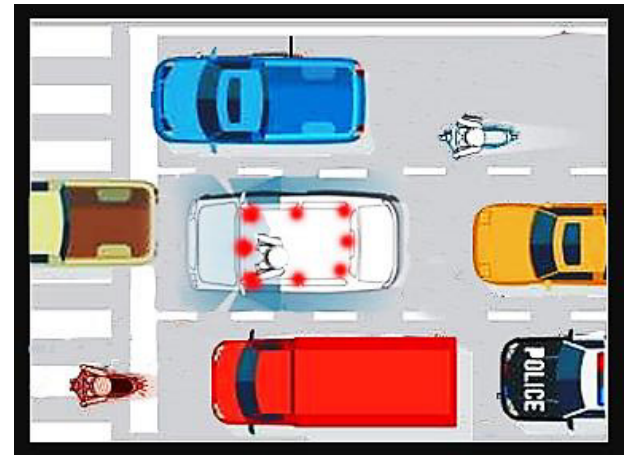

Figure 4. Audio source location in the $360^{\circ}$ proposal

Since 2016, there is a system in the market called Arkamys, which pretends to enhance security by changing the way alerts are emitted. The system uses as sources of sound the original car speakers to create the '3D-ADAS'. 


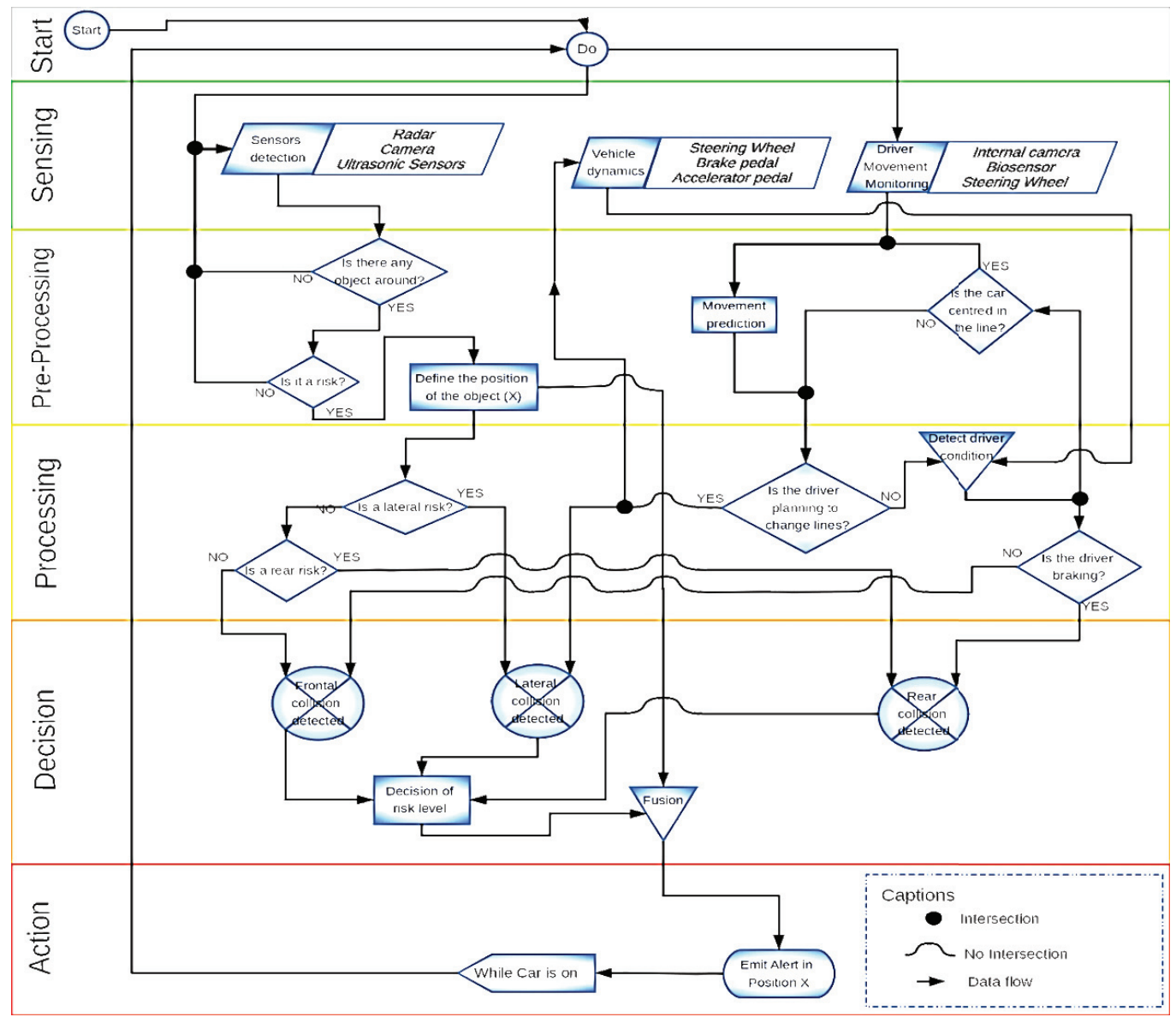

Figure 5. The general data flow diagram of proposed system

Despite it is a good concept, it has not been installed in any car yet, so, it is not clear how the system will be connected to ADAS and if necessary a major hardware and software integration.

Our proposal is to create a system like Arkamys, but smarter, means that we propose to use a 360-sound system inside the vehicle, which emits punctual sound source alerts based on prediction of driver movements and detection of hazards around the vehicle.

\section{1 $360^{\circ}$ Sensing}

Nowadays, the automobiles begin to carry more sensors that allow us to know the environment of the vehicle, providing the user with greater safety when driving.

There is a great variety of these, for example, with the use of ultrasonic sensors, radars of near and far range. Now is possible to determine the distance of objects to the car by using cameras with Artificial Intelligence, it is possible to detect and recognize objects to classify and issue alerts accurately.

Everything above is technologically possible and available in a car, so in our proposal we are increasing the 'intelligence' by predicting the driver's movements and

avoiding annoyances to the driver by emitting sound alerts only when necessary. Depending on the equipment available in the car, this will be used to improve the accuracy of alerts to the driver.
The main problem in a 360 ADAS alerts is the lateral monitoring, in a crowd via, the car will be surrounded by many cars, so predicting driver's movements will allow us to detect an intention to change lane and assisting the driver efficiently.

In figure 4 , is the data flow diagram to understand the basic logic of this proposed system, it is important to indicate that we have omitted the exhaustive detail of each part of the system since it will be stablished according to the available equipment.

\subsection{Movement predictions}

To predict driver movements, there are some approaches propose to detect it, in this case as the main objective is to assist the driver in the lateral control, the most common methods are:

- Cameras

- Vehicle dynamic parameters

- Neuromuscular Dynamics

The use of cameras by Kondyli in [13] shows an approach for the use of range cameras to detect upper body posture and driver movement in the car cabin with a skeletal model that follows the driver's movements, this principle could be useful for our approach and it is noninvasive to the driver.

In the other hand is the method proposed by Angkititrakul et al. [8] to create a line departure 
prediction system (LDPS) that is based in driving signals calculating the time to line crossing (TLC), The method of predicting the TLC is to estimate vehicle path and road curve and then calculate the time before the intersection of the two trajectories using the following relationship:

$$
T L C=\frac{y_{l}}{v \sin \phi}
$$

Where $y_{l}$ is the lateral distance from the lane boundary to the vehicle's reference (in meters), $v$ is the vehicle speed (in meters per second), and $\sin \phi$ is the vehicle relative yaw angle (in radians).

Finally, another proposal implies neuromuscular data to predict driver movements with a high success rate using a Queuing Network based driver model [14]. The results from 16 subjects showed that the proposed model performs very well, and its performance is closer to driver's actions when a vehicle runs at a relatively high speed.

By using the information obtained by the ECU through its OBD II port, it would detect the steering wheel angle, speed of movement and the activation of turning lights among other alerts, that would help the cameras system, which would allow us to define a critical action during driving.

Based on the review, available technology and methods of lane assistance shown above, it is proposed to create an intelligent driver assistance system by emitting sound alerts only when necessary, avoiding annoyance, in a 360 -sound configuration.

\section{The proposed approach}

To start with the development, a sound system will be installed inside a car around the driver as was shown in figure 4 , a $360^{\circ}$ audio set would be prepared to test different kinds of alerts that will help drivers detect the location of hazards around him even without seeing them.

The sound set in 360 would have alerts based on the configuration presented in the previous section by the NHTSA investigation [4], this would help us determine the effectiveness of this type of alerts in a 360 -sound environment selecting only a few audio types.

In addition, the vehicle dynamics would be recorded to generate a mathematical model to predict the driver's movements, as one of the presented in the previous section and it will depend of the instrumentation installed in the car when test is executed.

We firmly believe that as autonomous cars reach everyday life, it is important to develop systems to help humans in driving tasks and avoid traffic accidents that means high monetary costs and mainly lost lives.

\section{References}

1. Y. Zhang, C. Wu, and J. Wan, "Mathematical Modeling of the Effects of Speech Warning Characteristics on Human Performance and Its Application in Transportation Cyberphysical Systems," pp. 1-13, 2016.
2. F. Biondi, D. L. Strayer, R. Rossi, M. Gastaldi, and C. Mulatti, "Advanced driver assistance systems: Using multimodal redundant warnings to enhance road safety," Appl. Ergon., vol. 58, pp. 238-244, Jan. 2017.

3. J. Izquierdo-Reyes, R. A. Ramirez-Mendoza, and M. R. Bustamante-Bello, "A study of the effects of advanced driver assistance systems alerts on driver performance,” Int. J. Interact. Des. Manuf., Jan. 2017.

4. J. Singer, N. Lerner, C. Baldwin, and E. Traube, "Auditory Alerts in Vehicles: Effects of Alert Characteristics and Ambient Noise Conditions on Perceived Meaning and Detectability," ... Conference on the ..., vol. 1, pp. 1-14, 2015.

5. C. L. Lewis, B. A., Penaranda, B. N., Roberts, D. M., \& Baldwin, "Effectiveness of bimodal versus unimodal alerts for distracted drivers," Hum. Factors Driv. Assessment, Train. Veh. Des., pp. 376-382, 2013.

6. R. Kawamura, M. S. Bhuiyan, H. Kawanaka, and K. Oguri, "Simultaneous stimuli of vibration and audio for in-vehicle driver activation," IEEE Conf. Intell. Transp. Syst. Proceedings, ITSC, pp. 1710-1715, 2011.

7. E. E. Wiese and J. D. Lee, "Auditory alerts for invehicle information systems: the effects of temporal conflict and sound parameters on driver attitudes and performance.," Ergonomics, vol. 47, no. 9, pp. 965986, 2004.

8. P. Angkititrakul, R. Terashima, and T. Wakita, "On the Use of Stochastic Driver Behavior Model in Lane Departure Warning," IEEE Trans. Intell. Transp. Syst., vol. 12, no. 1, pp. 174-183, Mar. 2011.

9. F. Meng, S. Li, L. Cao, Q. Peng, M. Li, C. Wang, and W. Zhang, "Designing Fatigue Warning Systems: The perspective of professional drivers," Appl. Ergon., vol. 53, pp. 122-130, 2016.

10. F. Biondi and L. Skrypchuk, "Use Your Brain ( and Light ) for Innovative Human-Machine Interfaces," pp. 99-105, 2017.

11. V. Design, "In-vehicle noise alters the perceived meaning of auditory signals," pp. 401-407.

12. J. Izquierdo-Reyes, R. A. Ramirez-Mendoza, M. R. Bustamante-Bello, S. Navarro-Tuch, and R. AvilaVazquez, "Advanced driver monitoring for assistance system (ADMAS)," Int. J. Interact. Des. Manuf., Sep. 2016.

13. A. Kondyli, V. P. Sisiopiku, L. Zhao, and A. Barmpoutis, "Computer Assisted Analysis of Drivers' Body Activity Using a Range Camera," Intell. Transp. Syst. Mag. IEEE, vol. 7, no. 3, pp. 18-28, 2015.

14. L. Bi, M. Wang, C. Wang, and Y. Liu, "Development of a Driver Lateral Control Model by Integrating Neuromuscular Dynamics Into the Queuing Network-Based Driver Model," IEEE Trans. Intell. Transp. Syst., vol. 16, no. 5, pp. 2479-2486, Oct. 2015. 